\title{
Asymptotische Entwicklungen der Laguerreschen Polynome
}

\section{Doctoral Thesis}

Author(s):

Moecklin, Egon

Publication date:

1934

Permanent link:

https://doi.org/10.3929/ethz-a-000092417

Rights / license:

In Copyright - Non-Commercial Use Permitted 


\section{Asymptotische Entwicklungen der Laguerreschen Polynome}

Von der

Eidgenössischen Technischen Hochschule in Zürich zur Erlangung der Würde eines Doktors der Mathematik genehmigte

Promotionsarbeit vorgelegt von Egon Moecklin aus Diessenhofen (Thurgau)

Referent: Herr Prof. Dr. M. Plancherel Korreferent: Herr Prof. Dr. G. Pólya

$\begin{array}{llll}1 & 9 & 3 & 4\end{array}$ Art. Institut Orell Füssli, Zürich 
Separatabdruck aus dem Comment. math. helv. Bd. 7, S. 24 (1934/35) 


\section{Asymptotische Entwicklungen der Laguerreschen Polynome}

Von E. Moeckin, Zürich

\section{Einleitung}

§1. In der vorliegenden Arbeit werden asymptotische Entwicklungen für die Laguerreschen Polynome

$$
L_{n}(x)=e^{x} \cdot \frac{d^{n}}{d x^{n}}\left(x^{n} e^{-x}\right)=n ! \sum_{\nu=0}^{n}(-1)^{\nu}\left(\begin{array}{l}
n \\
v
\end{array}\right) \frac{x^{\nu}}{\nu !}
$$

aufgestellt. Wir beschränken uns auf die Betrachtung reeller Werte von $x$, behandeln aber sowohl den Fall $n|x| \rightarrow \infty$ wie den Fall, wo $n|x|$ beschränkt bleibt. Die angewandte Methode ist die Paßmethode, die in einer ähnlichen Untersuchung von M. Plancherel und W. Rotach ${ }^{1}$ ) zur Herleitung asymptotischer Entwicklungen der Hermiteschen Polynome gedient hat.

Aus (1) erhält man mit Hilfe der Cauchyschen Integralformel folgende Integraldarstellung von $L_{n}(x)$ :

$$
\frac{L_{n}(x)}{n !}=\frac{1}{2 \pi i} \cdot \int \frac{y^{n} e^{-(y-x)}}{(y-x)^{n+1}} d y=\frac{1}{2 \pi i} \int e^{-z}\left(1+\frac{x}{z}\right)^{n} \frac{d z}{z}=\frac{1}{2 \pi i} \int F(z) \frac{d z}{z},
$$

wo zur Abkürzung

$$
F(z)=e^{-z}\left(1+\frac{x}{z}\right)^{n}
$$

gesetzt ist. In der $z$-Ebene ist das Integral im positiven Sinn über eine einfach geschlossene Kurve zu erstrecken, die den Punkt $z=0$ im Innern enthält.

Die Funktion $F^{\prime}(z)$ besitzt außer $z=-x$ die beiden Nullstellen

$$
z=-\frac{x}{2} \pm \sqrt{\frac{x^{2}}{4}-n x}
$$

nämlich die Wurzeln der Gleichung $z^{2}+x z+n x=0$.

1) M. Plancherel et W. Rotach, Sur les valeurs asymptotiques des polynomos d'Hermite. Comment. math. helv. 1, 1929, p. 227-254. In Formel (12.) dieser Arbeit ist $\left(\frac{x}{2}\right)^{\frac{2 \nu}{3}}$ statt $\left(\frac{x}{2}\right)^{\frac{\nu}{3}} \mathrm{zu}$ lesen. 
Ist $x \neq 0,4 n$, so sind diese Wurzeln voneinander verschieden. Im Fall $0<x<4 n$ bezeichne $z_{0}$ diejenige mit positivem Imaginärteil, $z_{1}$ sei die konjugierte. In den Fällen $x>4 n$ und $x<0$ werde von den beiden reellen Wurzeln diejenige mit dem kleinern absoluten Betrag mit $z_{0}$ bezeichnet. Betrachten wir in der $z$-Ebene die Umgebung eines der Punkte (4), etwa $z_{0}$. Sie wird durch die Kurve $|F(z)|=\left|F\left(z_{0}\right)\right|$, für welche $z_{0}$ ein Doppelpunkt ist, in 4 Sektoren geteilt, in denen abwechselnd $|F(z)|>\left|F\left(z_{0}\right)\right|$ und $|F(z)|<\left|F\left(z_{0}\right)\right|$ ist. Die Fläche $w=|F(z)|$ besitzt für $z=z_{0}$ einen $\mathrm{Pa}$. Dasselbe gilt für $z=z_{1}$. Je nach dem zu betrachtenden Fall wählt man nun den Integrationsweg durch einen oder beide Pässe in den Sektoren, wo $|F(z)|<\left|F\left(z_{0}\right)\right|,\left|F^{\prime}(z)\right|<\left|F^{\prime}\left(z_{1}\right)\right|$ ist, so daß im $\mathrm{Pa}$ die Tangente an die Integrationskurve mit der Winkelhalbierenden des Sektors zusammenfällt. (Richtung des größten Gefälles von $|F(z)|$ ). Den wesentlichen Beitrag zum Integral (2) liefern dann die Umgebungen der Pässe, wie im folgenden gezeigt wird.

Ist $x=4 n$, so fallen $z_{0}$ und $z_{1}$ im Punkt $-\frac{x}{2}$ zusammen, dessen Umgebung in 6 Sektoren der beschriebenen Art geteilt wird.

\section{§ 2. Die asymptotischen Entwicklungen}

Die Polynome

$$
\varphi_{\lambda}(\xi)=\sum_{\mu=0}^{\lambda} a_{\lambda \mu} \xi^{\mu}, \chi_{\lambda p}(\xi)=\sum_{\mu=0}^{\lambda} b_{\lambda \mu}^{(p)} \xi^{\mu}, \psi_{\lambda}(\xi)=\sum_{\mu=0}^{\lambda} c_{\lambda \mu} \xi^{\mu} \quad(5,6,7)
$$

seien durch folgende Entwicklungen definiert:

$$
\begin{gathered}
\frac{e^{\xi \vartheta(\xi)}}{1+\zeta}=\sum_{\lambda=0}^{\infty} \phi_{\lambda}(\xi) \zeta^{\lambda}, \text { wo } g(\zeta)=\frac{\zeta}{1+\zeta}-\frac{z_{0}}{n} \sum_{\nu=3}^{\infty} \frac{1}{\nu}\left(\frac{z_{0}}{n} \zeta\right)^{\nu-2} ; \\
\frac{\left[h_{1}(\vartheta)\right]^{p}}{1-\vartheta} e^{\xi h_{2}(\vartheta)}=\sum_{\lambda=0}^{\infty} \chi_{\lambda p}(\xi) \vartheta^{\lambda}, \text { wo } h_{1}(\vartheta)=\sum_{\nu=1}^{\infty} \frac{1}{\nu}(2 \vartheta)^{v-1} \\
\text { und } h_{2}(\vartheta)=\frac{\vartheta}{1-\vartheta}-4 \sum_{\nu=4}^{\infty} \frac{1}{\nu}(2 \vartheta)^{\nu-3}
\end{gathered}
$$

$\exp \left[\xi \sum_{\nu=2}^{\infty} \frac{1}{\nu} \zeta^{\nu-1}\right]=\sum_{\lambda=0}^{\infty} \psi_{\lambda}(\xi) \zeta^{\lambda}$

Die folgenden 5 Fälle sind zu unterscheiden: 
I. $0<x<4 n ; n x \rightarrow \infty, n \rightarrow \infty$.

Definiert man den Winkel $a$ durch

$$
\cos \alpha=\frac{1}{2} \sqrt{\frac{x}{n}}\left(0<\alpha<\frac{\pi}{2}\right)
$$

so ergibt sich

$$
\begin{aligned}
\frac{L_{n}(x)}{n !}=\frac{(-1)^{n} e^{2 n \cos ^{2} \alpha}}{\pi \sqrt{n \sin 2 \alpha}}\left[\sum_{\lambda=0}^{k-1} \sum_{\mu=0}^{\lambda} r_{\lambda \mu} \frac{1+(-1)^{\lambda}}{2} \frac{\Gamma\left(\frac{\lambda+1}{2}+\mu\right)}{(\sin \alpha)^{\frac{\lambda}{2}+\mu}(2 n \cos \alpha)^{\frac{\lambda}{2}}}\right. \\
\times \cos \left\{n(2 \alpha-\sin 2 \alpha)-(\lambda+1) \frac{\pi}{4}+\alpha-\left(\frac{\pi}{2}-\alpha\right)(\lambda+\mu)+\alpha_{\lambda \mu}\right\} \\
+\mathrm{O}\left\{(\sin \alpha \sqrt{n \sin 2 \alpha})^{-k}\right\},
\end{aligned}
$$

wo $r_{\lambda \mu}$ und $\alpha_{\lambda, \mu}$ durch $a_{\lambda, \mu}=r_{\lambda_{i}} e^{i \omega} \lambda_{\mu \mu}$ bestimmt sind. Die Koeffizienten $a_{\lambda, \mu}$ sind von $\frac{z_{0}}{n}=-2 \cos \alpha e^{-i x}$ abhängig.

Ihre ersten Werte sind:

$$
\begin{aligned}
a_{00}=1 ; & \\
a_{20}=1, a_{21}= & \left.-2+\frac{4}{3} \cos ^{2} \alpha \cos 2 \alpha+2 \cos ^{3} \alpha \cos 3 \alpha\right) \\
& -i\left(\frac{4}{3} \cos ^{2} \alpha \sin 2 \alpha+2 \cos ^{3} \alpha \sin 3 \alpha\right), \\
a_{22}= & \left(\frac{1}{2}-\frac{4}{3} \cos ^{2} \alpha \cos 2 \alpha+\frac{8}{9} \cos ^{4} \alpha \cos 4 \alpha\right) \\
& +i\left(\frac{4}{3} \cos ^{2} \alpha \sin 2 \alpha-\frac{8}{9} \cos ^{4} \alpha \sin 4 \alpha\right)
\end{aligned}
$$

Die Koeffizienten $a_{\lambda \mu}$ mit ungeradem $\lambda$ braucht man nicht zu berechnen, da die entsprechenden Glieder in (11) verschwinden ${ }^{2}$ ).

II. $\boldsymbol{x}>$ 4n. Definiert man $\beta$ durch

$$
\operatorname{ch} \beta=\frac{1}{2} \sqrt{\frac{x}{n}} \quad(0<\beta<\infty),
$$

2) Für $k=3$ hat $W$. Rotach, eine asymptotische Formel für $L_{n}(x)$ angegeben: R $\operatorname{\theta ih} \Theta \mathbf{n}$ entwicklungen einer willkürlichen Funktion nach Hermiteschen und Laguerreschen Polynomen. Diss. E. T. H., Zürich 1925, S. 26. 
so ergibt sich

$$
\begin{gathered}
\frac{L_{n}(x)}{n !}=\frac{(-1)^{n}(\operatorname{ch} 2 \beta+\operatorname{sh} 2 \beta)^{n} e^{2 n \operatorname{ch} \beta(\operatorname{ch} \beta-s h \beta)}}{2 \pi \sqrt{n \operatorname{sh} 2 \beta}(\operatorname{ch} \beta-\operatorname{sh} \beta)}\left[\sum_{\lambda=0}^{k-1} \sum_{\mu=0}^{\lambda} a_{\lambda, \mu} \frac{1+(-1)^{\lambda}}{2}\right. \\
\left.\quad \times \frac{(-1)^{\frac{\lambda}{2}} \Gamma\left(\frac{\lambda+1}{2}+\mu\right)}{(\operatorname{sh} \beta)^{\frac{\lambda}{2}+\mu}}(2 n \operatorname{ch} \beta)^{\frac{\lambda}{2}}(\operatorname{ch} \beta-\operatorname{sh} \beta)^{\lambda+\mu}+O\left\{n^{-\frac{k}{2}}(\operatorname{th} \beta)^{-\frac{3 k}{2}}\right\}\right]
\end{gathered}
$$

Die Koeffizienten $a_{\lambda \mu}$ sind von $\frac{z_{0}}{n}=-\frac{\left|z_{0}\right|}{n}=-2 \operatorname{ch} \beta(\operatorname{ch} \beta-\operatorname{sh} \beta)$ abhängig. Ihre ersten Werte sind:

$$
\begin{aligned}
a_{00}=1 ; & 1, a_{21}=-2+\frac{4}{3} \operatorname{ch}^{2} \beta(\operatorname{ch} \beta-\operatorname{sh} \beta)^{2}+2 \operatorname{ch}^{3} \beta(\operatorname{ch} \beta-\operatorname{sh} \beta)^{3}, \\
a_{20}=1 & \frac{1}{2}\left[1-\frac{4}{3} \operatorname{ch}^{2} \beta(\operatorname{ch} \beta-s h \beta)^{2}\right]^{2} .
\end{aligned}
$$

III. $\boldsymbol{x}<0$; $\boldsymbol{n}|\boldsymbol{x}| \rightarrow \infty, \boldsymbol{n} \rightarrow \infty$.

Die Einführung der Hilfsgröße $\gamma$ durch

$$
s h^{2}(2 \gamma)=-\frac{4 n}{x}(0<\gamma<\infty)
$$

ergibt:

$$
\begin{array}{r}
\frac{L_{n}(x)}{n !}=\frac{e^{\frac{n}{c^{2} \gamma}}(c t h \gamma)^{2 n} c h^{2} \gamma}{\pi \sqrt{2 n c h 2 \gamma}}\left[\sum_{\lambda=0}^{k-1} \sum_{\mu=0}^{\lambda} a_{\lambda_{\mu}} \frac{1+(-1)^{\lambda}}{2} \Gamma\left(\frac{\lambda+1}{2}+\mu\right)\right. \\
\left.\quad \times \frac{(-1)^{\frac{\lambda}{2}} 2^{\frac{\lambda}{2}+\mu}(c h \gamma)^{2(\lambda+\mu)}}{n^{\frac{\lambda}{2}}(c h 2 \gamma)^{\frac{\lambda}{2}+\mu}}+0\left\{n^{-\frac{k}{2}}(c h \gamma)^{k}\right\}\right]
\end{array}
$$

Die ersten Koeffizienten $a_{\lambda, \mu}$ sind:

$$
\begin{gathered}
\left(\frac{z_{0}}{n}=-\frac{\left|z_{0}\right|}{n}=-\frac{1}{c h^{2} \gamma}\right) \\
a_{00}=1 ; \\
a_{20}=1, a_{21}=-2+\frac{1}{c h^{4} \gamma}\left(\frac{1}{3}+\frac{1}{4 c h^{2} \gamma}\right), a_{22}=\frac{1}{2}\left(1-\frac{1}{3 c h^{4} \gamma}\right)^{2} .
\end{gathered}
$$


IV. $\boldsymbol{x} \sim \mathbf{4 n}$. Führen wir die Hilfsgröße

$$
t=2\left(\frac{x}{2}\right)^{-\frac{1}{3}} \cdot\left(n-\frac{x}{4}\right)
$$

ein. Es lassen sich 2 positive Konstanten $d_{1}$ und $d_{2}$ finden, so daß für $|t| .<d_{1} x^{\frac{2}{3}}$ gilt:

$$
\begin{aligned}
\frac{L_{n}(x)}{n !}= & \frac{(-1)^{n} e^{\frac{x}{2}}}{\pi\left(\frac{x}{2}\right)^{\frac{1}{3}}}\left[\sum_{p=0}^{\infty} \frac{A_{p}(x)}{p !} t^{p}+0\left(x^{\frac{1}{3}} e^{-d_{2} x}\right)\right] \\
A_{p}(x)=(-1)^{p} 3^{\frac{p-2}{3}} \sum_{\lambda=0}^{k-1} \sum_{\mu=0}^{\lambda} \frac{3^{\frac{2}{3}+\mu}}{\Gamma\left(\frac{p+\lambda+1}{3}+\mu\right)} & \left(\frac{x}{2}\right)^{\frac{\lambda}{3}} \\
& \times b_{\lambda \cdot u}^{(p)} \sin \left(\frac{2(p+\lambda+1)}{3} \pi\right)+0\left(x^{-\frac{k}{3}}\right) .
\end{aligned}
$$

Die ersten Koeffizienten $b_{\lambda, u}^{(p)}$ sind:

$b_{00}^{(p)}=1$;

$b_{10}^{(p)}=p+1, b_{11}^{(p)}=-1 ;$

$b_{30}^{(p)}=\frac{3 p^{2}+11 p}{6}+1, b_{21}^{(p)}=-\left(p+\frac{16}{5}\right), b_{22}^{(p)}=\frac{1}{2}$.

Die in (14) auftretende Reihe ist eine ganze Funktion von $t$. Vernachlässigt man in (14) die Glieder von einem gewissen $p$ an, so läßt sich der Fehler abschätzen $\left.{ }^{3}\right)$.

V. $n|x|$ beschränkt, $n \rightarrow \infty$.

Bezeichnet man die Besselsche Funktion $v$. Ordnung mit $J_{v}$, so ergibt sich $\frac{L_{n}(x)}{n !}=\sum_{\lambda=0}^{k-1} \sum_{\mu=0}^{\lambda}(-1)^{\mu} c_{\lambda \mu} n^{-\frac{\lambda-\mu}{2}} x^{\frac{\lambda+\mu}{2}} J_{\lambda+\mu}(2 \sqrt{n x})+\mathrm{O}\left(n^{-k}\right)$.

$\left.{ }^{3}\right)$ Vergleiche $M$. Plancherel und $W$, Rotach a. a. O. § 16. 
Für $k=1,2,3$ erhalten wir insbesondere:

$$
\begin{aligned}
\frac{L_{n}(x)}{n !} & =J_{0}(2 \sqrt{n x})+\mathrm{O}\left(\frac{1}{n}\right) \\
& =J_{0}(2 \sqrt{n x})-\frac{x}{2} J_{2}(2 \sqrt{n x})+\mathrm{O}\left(\frac{1}{n^{2}}\right) \\
& =J_{0}(2 \sqrt{n x})-\frac{x}{2} J_{2}(2 \sqrt{n x})-\frac{1}{3} n^{-\frac{1}{2} x^{\frac{3}{2}} J_{3}(2 \sqrt{n x})} \\
& +\frac{x^{2}}{8} J_{4}(2 \sqrt{n x})+\mathrm{O}\left(\frac{1}{n^{3}}\right) .
\end{aligned}
$$

Ist $n$ beschränkt und $|x| \rightarrow \infty$, so gilt nach (1)

$$
\begin{aligned}
L_{n}(x)=(-1)^{n} x^{n}\left[1-\left(\begin{array}{l}
n \\
1
\end{array}\right)^{2} \frac{1}{x}+2 !\left(\begin{array}{l}
n \\
2
\end{array}\right)^{2} \frac{1}{x^{2}}+\ldots\right. \\
\left.+(-1)^{k-1}(k-1) !\left(\begin{array}{c}
n \\
k-1
\end{array}\right)^{2} \frac{1}{x^{k-1}}+0\left(\frac{1}{x^{k}}\right)\right](k \leqq n) .
\end{aligned}
$$

Die Anwendung der Formeln (11) - (15) setzt voraus, daß $n$ groß ist. In Formel (11) zeigt dies der Ausdruck

$$
\sin \alpha \sqrt{n \sin 2 \alpha}=(n x)^{\frac{1}{4}} \cdot\left(1-\frac{x}{4 n}\right)^{\frac{3}{4}}=\frac{1}{2} \sqrt{\frac{x}{n}} t^{\frac{3}{4}} .
$$

Ist $n$ groß, nicht aber $\sin a \sqrt{n \sin \overline{2} \alpha}$, so ist entweder $n x$ beschränkt oder $\frac{x}{4 n}$ liegt in der Nähe von 1. Im ersten Fall ist die Formel (15), im zweiten Fall die Formel (14) zu verwenden.

Die Formel (12) ist zu benutzen, wenn der Ausdruck

$$
n^{\frac{1}{2}}(t h \beta)^{\frac{3}{2}}=n^{\frac{1}{2}}\left(1-\frac{4 n}{x}\right)^{\frac{3}{4}}=\sqrt{\frac{2 n}{x}}|t|^{\frac{3}{4}}
$$

groß ist. Ist $n$ groß (und damit auch $x$ ), nicht aber $n^{\frac{1}{2}}(t h \beta)^{\frac{3}{2}}$, so liegt $\frac{4 n}{x}$ in der Nähe von 1, und man gebraucht die Formel (14). 
Die Formel (13) ist brauchbar, wenn der Ausdruck

$$
\frac{n}{c h^{2} \gamma}=\frac{2}{\frac{1}{n}+\sqrt{\frac{1}{n^{2}}-\frac{4}{n x}}}
$$

groß ist. Ist $n$ groß, nicht aber $\frac{n}{c h^{2} \gamma}$, so ist $n|x|$ beschränkt, und man verwendet die Formel (15).

\section{Kapitel.}

$$
\text { Der Fall } 0<x<4 n ; n x \rightarrow \infty, n \rightarrow \infty \text {. }
$$

§3. Wir wählen den Integrationsweg symmetrisch zur reellen Axe der $z$-Ebene durch die beiden Punkte $z_{0}$ und $z_{1}$. In der oberen Halbebene setzt er sich aus 3 Kreisbogenstücken $C_{0}, C_{2}$ und $C_{3}$ zusammen (Fig. 1), deren genaue Festlegung später erfolgen wird. Da die Beiträge der symmetrischen Kurventeile in der oberen und unteren Halbebene vom Integral (2) konjugiert ausfallen, kann man sich auf den ersten Teil beschränken. Der Beitrag der Umgebung von $z_{0}$ soll zunächst bestimmt werden.

\section{§ 4. Umgebung des Passes $z_{0}$.}

Die Substitution $\frac{1}{z}=\frac{1+\zeta}{z_{0}}$ ergibt

$$
\frac{F(z)}{F\left(z_{0}\right)}=\exp \left[\frac{z_{0} \zeta}{1+\zeta}+n l g\left(1-\frac{z_{0}}{n} \zeta\right)\right] .
$$

Für $|\zeta|<\frac{n}{\left|z_{0}\right|}$ und, da

$$
\left|z_{0}\right|=\sqrt{n x}, \frac{n}{\left|z_{0}\right|}=\sqrt{\frac{n}{x}}>\frac{1}{2},
$$

a fortiori für $|\zeta| \leqq \frac{1}{2}$ erhält man die Entwicklung

$$
\frac{F(z)}{F\left(z_{0}\right)}=\exp \left[-a z_{0} \zeta^{2}+\frac{z_{0} \zeta^{3}}{1+\zeta}-\frac{z_{0}^{2} \zeta^{2}}{n} \sum_{\nu=3}^{\infty} \frac{1}{\nu}\left(\frac{z_{0}}{n} \zeta\right)^{v-2}\right]
$$




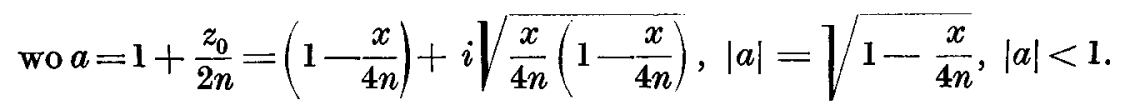

Man findet jetzt

$$
F(z) \cdot \frac{d z}{z}=-F\left(z_{0}\right) e^{-a z_{0} \zeta^{2}} \cdot \frac{e^{\xi \theta(\zeta)}}{1+\zeta} d \zeta
$$

wo $\xi=z_{0} \zeta^{2}$ und $g(\zeta)$ durch $\left(8^{\prime}\right)$ gegeben ist.

Setzt $\operatorname{man} \zeta=\frac{u}{\sqrt{a z_{0}}}$, so ist $u$ reell für die Richtung des größten Gefälles im Paß $z_{0}$, dem der Punkt $u=0$ entspricht. In der $u$-Ebene erstrecken wir das Integral von

$$
u_{2} \text { bis } u_{3} \text {, wo }-u_{2}=u_{3}=\Theta \cdot \frac{\left|\sqrt{a z_{0}}\right|}{2}, \quad 0<\Theta<1 .
$$

Den diesem Wegstück entsprechenden Kreisbogen der z-Ebene bezeichnen wir mit $C_{0}$, seine Endpunkte mit $z_{2}$ und $z_{3}$. Führen wir in (17) die Entwicklung (8) ein, so resultiert für den Beitrag von $C_{0}$ :

$$
\frac{1}{2 \pi i} \int_{C_{0}} F(z) \frac{d z}{z}=-\frac{F\left(z_{0}\right)}{2 \pi i \sqrt{a z_{0}}}\left[\sum_{\lambda=0}^{k-1} \int_{u_{2}}^{u_{3}} e^{-u^{2}} p_{\lambda}\left(\frac{u^{2}}{a}\right)\left(\frac{u}{\sqrt{a z_{0}}}\right)^{\lambda} d u+R_{k}^{\prime}\right] \text {, }
$$

wenn zur Abkürzung

$$
R_{k}^{\prime}=\int_{u_{2}}^{u_{3}} e^{-u^{2}} \sum_{\lambda=k}^{\infty} \varphi_{\lambda}\left(\frac{u^{2}}{a}\right)\left(\frac{u}{\sqrt{a z_{0}}}\right)^{\lambda} d u
$$

gesetzt ist. Erstreckt man in der endlichen Summe die Integration von $-\infty$ bis $+\infty$, so begeht man einen Fehler $R_{k}^{\prime \prime}$ und man erhält:

$$
\begin{aligned}
\frac{1}{2 \pi i} \int_{C_{0}} F(z) \frac{d z}{z}=-\frac{F\left(z_{0}\right)}{2 \pi i \sqrt{a z_{0}}} & \sum_{\lambda=0}^{k-1} \sum_{\mu=0}^{\lambda} a_{\lambda_{\mu}} a-\left(\frac{\lambda}{2}+\mu\right) z_{z_{0}}-\frac{\lambda}{2} \frac{1+(-1)^{\lambda}}{2} \\
& \left.\times \Gamma\left(\frac{\lambda+1}{2}+\mu\right)+R_{k}^{\prime}+R_{k}^{\prime \prime}\right] .
\end{aligned}
$$

Die Koeffizienten $a_{\lambda_{\mu}}$ sind durch (5) und (8) bestimmt. 
§ 5. Abschätzung von $\boldsymbol{R}_{\boldsymbol{k}}^{\prime}$ und $\boldsymbol{R}_{\boldsymbol{k}}^{\prime \prime}$.

Die Funktion $g(\zeta)\left(8^{\prime}\right)$ besitzt auf Grund von $\frac{\left|z_{0}\right|}{n}<2$ die Majorante $\sum_{\nu=1}^{\infty} \zeta^{\nu}+2 \sum_{\nu=3}^{\infty} \frac{1}{\nu}(2 \zeta)^{\nu-2}$, also a fortiori die Majorante

$$
\sum_{\nu=1}^{\infty} \frac{(2 \zeta)^{\nu}}{\nu}+2 \sum_{\nu=1}^{\infty} \frac{(2 \zeta)^{v}}{\nu}=-3 \lg (1-2 \zeta), \quad\left(|\zeta|<\frac{1}{2}\right) .
$$

Die Funktion (8) läßt daher die Majorante

$$
(1-2 \zeta)^{-(1+3|\xi|)}=1+\left(1+\frac{3|\xi|}{1}\right)(2 \zeta)+\left(1+\frac{3|\xi|}{1}\right)\left(1+\frac{3|\xi|}{2}\right)(2 \zeta)^{2}+\ldots
$$

zu. Daraus erhält man die Ungleichung

$$
\left|\sum_{\lambda=k}^{\infty} \varphi_{\lambda}(\xi) \zeta^{\lambda}\right| \leqq 2^{k}(1+3|\xi|)^{k}(1-2|\zeta|)^{-(1+3|\xi|)}|\zeta|^{k}
$$
Beachtet man, daß im Integrationsintervall $u_{2} \leqq u \leqq u_{3} \quad 1-2\left|\frac{u}{\sqrt{a z_{0}}}\right|$
$\geqq 1-\Theta$, so erhält man für $R_{k}^{\prime}$ die Abschätzung

$$
\left|R_{k}^{\prime}\right| \leqq\left(\frac{2}{\mid \sqrt{a z_{0}}}\right)^{k} \cdot \frac{1}{1-\Theta} \int_{u_{2}}^{u_{3}}\left[e(1-\Theta)^{\frac{3}{a}}\right]^{-u^{2}}\left(1+3\left|\frac{u^{2}}{a}\right|\right)^{k}|u|^{k} d u
$$

Hieraus findet man, falls $\Theta$ hinreichend klein gewählt wird,

$$
R_{k}{ }^{\prime}=\mathrm{O}\left(\left|a \sqrt{a z_{0}}\right|^{-k}\right)
$$

Das in (19) stehende Fehlerglied $R_{k}^{\prime \prime}$ kann unter der Voraussetzung $\left|a \sqrt{a z_{0}}\right| \rightarrow \infty$ gegenüber $R_{k}^{\prime}$ vernachlässigt werden ${ }^{4}$ ).

4) Vergleiche M. Plancherel und W. Rotach a. a. O., S. 238. 


\section{§6. Beitrag des übrigen Integrationsweges.}

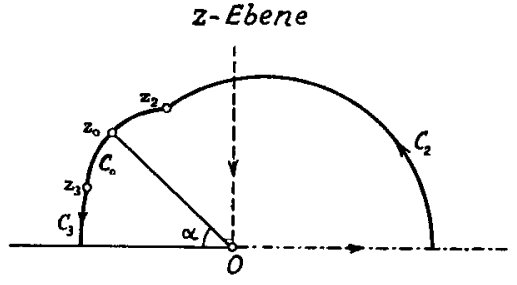

Fig.1
$\zeta$-Ebene

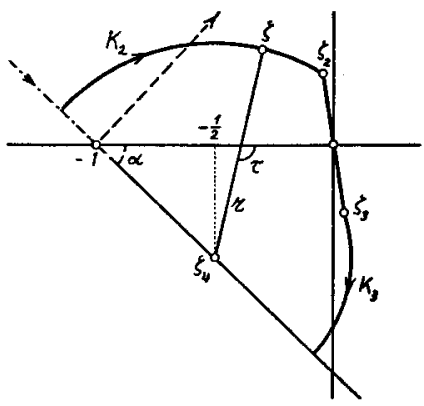

Fig. 2

Die den Punkten $u_{2}$ und $u_{3}$ der $u$-Ebene ( $\S$ 4) entsprechenden Punkte der $\zeta$-Ebene seien $\zeta_{2}$ und $\zeta_{3}$ (Fig. 2). Durch $\zeta_{2}$ legen wir einen Kreisbogen $K_{2}$, dessen Zentrum $\zeta_{4}$ die Abszisse $-\frac{1}{2}$ hat und auf der Geraden liegt, welche der reellen Axe der $z$-Ebene entspricht. Das Bild von $K_{2}$ in der $z$-Ebene nennen wir $C_{2}$. Den Beitrag von $C_{2}$ wollen wir zunächst abschätzen.

Vorerst ist zu zeigen, daß der gewählte Kreis den Punkt - 1 der $\zeta$-Ebene einschließt, d. h. daß $r>s$, wenn mit $r$ der Radius von $K_{2}$ und mit $s$ die Strecke $\left(-1, \zeta_{4}\right)$ bezeichnet wird.

Zur Bestimmung von $r$ gehen wir aus von $u=\zeta \sqrt{a z_{0}}$, woraus $\arg u_{2}=\arg \zeta_{2}+\frac{1}{2}\left(\arg a+\arg z_{0}\right)$. Führen wir den Winkel $\alpha$ ein durch $\arg z_{0}=\pi-\alpha(\alpha$ ist also mit dem in $\S 2$ I eingeführten Winkel identisch), so wird

$\operatorname{tg} \alpha=\sqrt{\frac{4 n}{x}-1}=\operatorname{ctg}(\arg a), \arg a=\frac{\pi}{2}-\alpha$, folglich

$\pi=\arg \zeta_{2}+\frac{1}{2}\left(\frac{3 \pi}{2}-2 \alpha\right)$, oder $\arg \zeta_{2}=\frac{\pi}{4}+\alpha$.

Somit ist $r^{2}=s^{2}+s\left|\zeta_{2}\right| \sqrt{2}+\left|\zeta_{2}\right|^{2}$ und daher $r>s$.

Für $s$ erhält man

$$
s=\frac{1}{2 \cos \alpha}=\sqrt{\frac{n}{x}}=\frac{n}{\left|z_{0}\right|} .
$$


Abgesehen vom Faktor $\frac{1}{2 \pi i}$ ergibt sich für den Beitrag von $C_{2}$ :

$$
\int_{\sigma_{2}} F(z) \frac{d z}{z}=-\int_{K_{2}} \exp \left[-\frac{z_{0}}{1+\zeta}\right]\left(1+\frac{x(1+\zeta)}{z_{0}}\right)^{n} \frac{d \zeta}{1+\zeta}=-\int_{K_{2}} H(\zeta) \frac{d \zeta}{1+\zeta} .
$$

Das Integral über $K_{\mathbf{2}}$ schreiben wir in der Form:

$\int_{K_{2}} H(\zeta) \frac{d \zeta}{1+\zeta}=H(o) \frac{H\left(\zeta_{2}\right)}{H(o)} \int_{K_{2}} \frac{H(\zeta)}{H\left(\zeta_{2}\right)} \frac{d \zeta}{1+\zeta}$, wobei

$\int_{\boldsymbol{K}_{2}} \frac{H(\zeta)}{H\left(\zeta_{2}\right)} \frac{d \zeta}{1+\zeta}=\int_{\boldsymbol{K}_{2}} \exp \left[\left|z_{0}\right| e^{-i \alpha}\left(\frac{1}{1+\zeta}-\frac{1}{1+\zeta_{2}}\right)\right]\left(\frac{\frac{n}{\left|z_{0}\right|} e^{i \alpha}+\zeta}{\frac{n}{\left|z_{0}\right|} e^{i \alpha}+\zeta_{2}}\right)^{n} \frac{d \zeta}{1+\zeta}$.

Auf $K_{2}$ setzen wir nun $\zeta=-1+s e^{-i \alpha}-r e^{-i \tau}=-s e^{i \alpha}-r e^{-i \tau}$, wo die Bedeutung von $\tau$ aus Fig. 2 ersichtlich ist.

Entspricht dem Punkt $\zeta_{2}$ der Winkel $\tau=\tau_{0}$, so folgt

$$
\frac{\frac{n}{\left|z_{0}\right|} e^{i \alpha}+\zeta}{\frac{n}{\left|z_{0}\right|} e^{i \alpha}+\zeta_{2}}=\frac{s e^{i \alpha}+\zeta}{s e^{i \alpha}+\zeta_{2}}=e^{i\left(\tau_{0}-\tau\right)} .
$$

Führen wir noch die folgenden abkürzenden Bezeichnungen ein :

$$
\Phi_{1}(\tau)=\Re\left[e^{-i \alpha}\left(\frac{1}{1+\zeta}-\frac{1}{1+\zeta_{2}}\right)\right], \Phi_{2}(\tau)=\frac{1}{|1+\zeta|},
$$

so erhält man

$$
\left|\int_{\boldsymbol{R}_{2}} \frac{H(\zeta)}{H\left(\zeta_{2}\right)} \frac{d \zeta}{1+\zeta}\right| \leqq r \int_{\alpha}^{\tau_{0}} \exp \left[\left|z_{0}\right| \Phi_{1}(\tau)\right] \Phi_{2}(\tau) d \tau=r \int_{\alpha}^{\tau_{0}} f(\tau) d \tau .
$$

Die Funktion $f(\tau)$ ist eine mit $\tau$ wachsende Funktion und nimmt den maximalen Wert für die obere Integrationsgrenze $\tau=\tau_{0}$ an. Die Rechnung liefert nämlich

$$
\Phi_{1}(\tau)=\frac{s-r \cos (\tau-\alpha)}{s^{2}+r^{2}-2 r s \cos (\tau-a)}-\Re\left(e^{-i \alpha} \frac{1}{1+\zeta_{2}}\right) \text { und }
$$




$$
f^{\prime}(\tau)=\Phi_{2}^{3} e^{\left|z_{0}\right| \Phi_{1}} r \cdot s \cdot \sin (\tau-\alpha)\left[\frac{\left|z_{0}\right|\left(r^{2}-s^{2}\right)}{s} \Phi_{2}^{2}-1\right]
$$

Nun ist $\frac{\left|z_{0}\right|\left(r^{2}-s^{2}\right)}{s}>\left|z_{0}\right|\left|\zeta_{2}\right| \sqrt{2} \rightarrow \infty \quad$ und folglich $f^{\prime}(\tau) \geqq 0$ $(=0$ für $\tau=a)$. Weiter erhält man $f\left(\tau_{0}\right)=\Phi_{2}\left(\tau_{0}\right)=\frac{1}{\left|1+\zeta_{2}\right|}<2$ und daher

$$
\left|\int_{K_{2}} \frac{H(\zeta)}{H\left(\zeta_{2}\right)} \frac{d \zeta}{\mathrm{I}+\zeta}\right|<2 r\left(\tau_{0}-\alpha\right)<2 r(\pi-2 \alpha)=4 r\left(\frac{\pi}{2}-\alpha\right) .
$$

Diese Abschätzung genügt noch, wenn $r$ sehr groß ist, d. h. wenn

$$
\frac{n}{\left|z_{0}\right|}=\sqrt{\frac{n}{x}} \text { sehr groß ist; denn }
$$

$4 r\left(\frac{\pi}{2}-\alpha\right)<\frac{4 r}{\operatorname{tg} \alpha} \sim 2$, weil $r \sim \sqrt{\frac{n}{x}}$ und $\operatorname{tg} \alpha=\sqrt{\frac{4 n}{x}-1} \sim 2 \sqrt{\frac{n}{x}}$.

Da

$$
H(0)=F\left(z_{0}\right) \text { und }\left|\frac{H\left(\zeta_{2}\right)}{H(o)}\right|<e^{-\delta\left|a z_{0}\right|},
$$

so ergibt sich schließlich

$$
\left|\frac{1}{2 \pi i} \int_{C_{2}} F(z) \frac{d z}{z}\right|<M_{1}\left|F\left(z_{0}\right)\right| e^{-\delta\left|a z_{0}\right|},
$$

wo $\delta$ und $M_{1}$ zwei positive Konstanten sind.

Wählt man durch den Punkt $\zeta_{3}$ den Kreisbogen $K_{3}$ mit demselben Mittelpunkt $\zeta_{4}$ wie für $K_{2}$, so findet man für den Beitrag über den entsprechenden Weg $C_{3}$ der $z$-Ebene eine analoge Abschätzung wie über $C_{2}$, vorausgesetzt $\operatorname{da} \beta \sqrt{\frac{n}{x}}$ beschränkt ist. Ist dies nicht der Fall, so ersetzen wir $K_{3}$ durch den Kreisbogen $K_{3}^{*}$, dessen Zentrum $\zeta_{4}^{*}$ auf der Mittelsenkrechten zur Verbindungsstrecke der beiden Punkte -1 und $\zeta_{3}$ liegt. (Fig. 3). Sein Bild in der $z$-Ebene nennen wir $C_{3}^{*}$. 


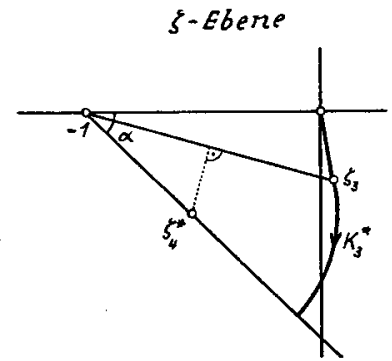

Fig.3

Das Integral über $K_{3}^{*}$ schreiben wir in der gleichen Form wie dasjenige über $K_{\mathbf{2}}$ und setzen

$\zeta=-1+r^{*}\left(e^{-i \hbar}-e^{-i t}\right)$, wo mit $r^{*}$ der Radius von $K_{3}^{*}$ bezeichnet ist.

Dann gilt auf $K_{3}^{*}$

$$
\Re\left[e^{i a}\left(\frac{1}{1+\zeta}-\frac{1}{1+\zeta_{3}}\right)\right]=0
$$

$$
\left|\frac{\frac{n}{\left|z_{0}\right|} e^{i \ell}+\zeta}{\frac{n}{\left|z_{0}\right|} e^{i \alpha}+\zeta_{3}}\right|^{n} \text { nimmt mit wachsendem } \tau \text { vom Wert } l \text { an ab. }
$$

Somit wird für eine geeignete positive Konstante $M_{2}$

$$
\left|\int_{K_{3}^{*}} \frac{\dot{H}(\zeta)}{H\left(\zeta_{3}\right)} \frac{d \zeta}{1+\zeta}\right|<M_{2} r^{*}
$$

Falls $\sqrt{\frac{n}{x}} \rightarrow \infty$ bleibt $r^{*}$ kleiner als $\frac{m}{\left|\zeta_{3}\right|}=\frac{1}{\delta^{\prime}}\left(m\right.$ und $\delta^{\prime}$ sind positive Konstanten), und es ergibt sich für den Beitrag von $C_{3}^{*}$

$$
\left|\frac{1}{2 \pi i} \int_{C_{3}^{\star}} F(z) \frac{d z}{z}\right|<M_{2}\left|F\left(z_{0}\right)\right| \frac{1}{\delta^{\prime}} e^{-\delta\left|a z_{0}\right|} .
$$

\section{§ 7. Zusammenfassung}

Die Abschätzungen (20), (21) und (22) zeigen, daß unter der Voraussetzung $\left|a \sqrt{a z_{0}}\right| \rightarrow \infty$ die Umgebung des Passes $z_{0}$ den wesentlichen in (19) enthaltenen Beitrag liefert. 
Die Einführung des wiederholt gebrauchten Winkels $\alpha$ ergibt die Ausdrücke

$$
\begin{aligned}
& z_{0}=-2 n \cos \alpha e^{-i \alpha},\left(1+\frac{x}{z_{0}}\right)^{n}=(-1)^{n} e^{2 i n \alpha}, \\
& a=\sin \alpha e^{i\left(\frac{\pi}{2}-\alpha\right)}, \sqrt{a z_{0}}=\sqrt{n \sin 2 \alpha} e^{i\left(\frac{3 \pi}{4}-\alpha\right)} .
\end{aligned}
$$

Fügt man zu (19) den vom $\mathrm{Paß} z_{1}$ herrührenden konjugierten Beitrag, so erhält man schließlich die Formel (11) der Einleitung.

\section{Kapitel. \\ Der Fall $x>4 n$.}

$\S 8$. Wir wählen den Integrationsweg symmetrisch zur reellen Axe der $z$-Ebene durch den einen $\mathrm{Pa}$

$$
z_{0}=-\frac{x}{2}+\sqrt{\frac{x^{2}}{4}-n x}
$$

Er setzt sich aus 2 Kreisbogenstücken $C_{0}$ und $C$ zusammen (Fig. 4), die wir später genauer festlegen werden. Mit einigen Abweichungen sind die Ausführungen des vorhergehenden Kapitels übertragbar.

\section{§ 9. Umgebung des Passes.}

Beachtet man die Beziehungen

$$
\begin{aligned}
& \left|z_{0}\right|=-z_{0}=\frac{2 n}{1+\sqrt{1-\frac{4 n}{x}}}, n<\left|z_{0}\right|<2 n, \\
& 0<a=1+\frac{z_{0}}{2 n}<\frac{1}{2},
\end{aligned}
$$

so ergibt sich für den Beitrag der Umgebung von $z_{0}$

$$
\begin{array}{r}
\frac{1}{2 \pi i} \int_{C_{0}} F^{\prime}(z) \frac{d z}{z}=\frac{F}{2 \pi \sqrt{n})} \sqrt{\sqrt{a\left|z_{0}\right|}}\left[\sum_{\lambda=0}^{k-1} \sum_{\mu=0}^{\lambda} i^{\lambda} a_{\lambda \mu} a^{-\left(\frac{\lambda}{2}+\mu\right)}\left|z_{0}\right|^{-\frac{\lambda}{2}}\right. \\
\left.\times \frac{1+(-1)^{\lambda}}{2} \Gamma\left(\frac{\lambda+1}{2}+\mu\right)+R_{k}^{\prime}+R_{k}^{\prime \prime}\right] .
\end{array}
$$


Hier sind die Koeffizienten $a_{\lambda \mu}$ wieder durch (5) und (8) bestimmt; die Bedeutung von $C_{0}, R_{k}^{\prime}$ und $R_{k}^{\prime \prime}$ ist analog wie im $\S 4$.

Da auch in diesem Fall $\frac{\left|z_{0}\right|}{n}<2$, so gilt die Abschätzung von $R_{k}^{\prime}$ im $\S 5$ hier unverändert; $R_{k}^{\prime \prime}$ kann unter der Voraussetzung $a \sqrt{a\left|z_{0}\right|} \rightarrow \infty$ gegenüber $R_{k}^{\prime}$ vernachlässigt werden.

\section{$\S$ 10. Beitrag des übrigen Integrationsweges}

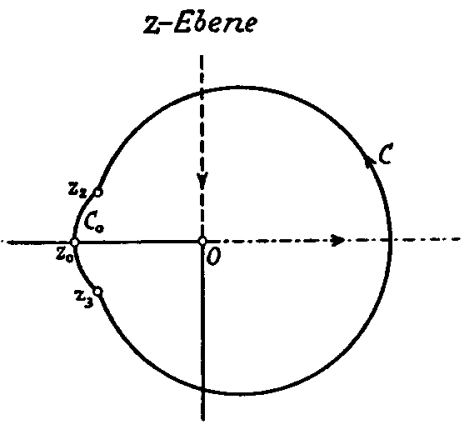

Fig. 4

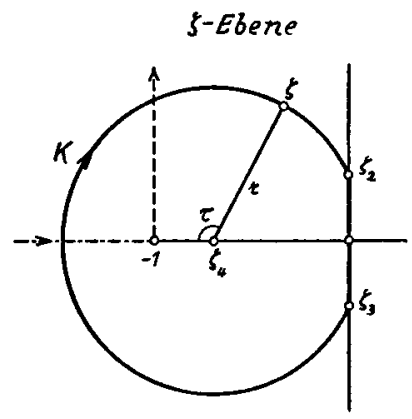

Fig. 5

Durch die Endpunkte $\zeta_{2}$ und $\zeta_{3}$ des geradlinigen Wegstückes der $\zeta$-Ebene legen wir einen Kreisbogen $K$ mit dem Zentrum $\zeta_{4}=\frac{n}{\left|z_{0}\right|}=-s$.

Der entsprechende Kreisbogen der $z$-Ebene sei $C$. Ist $r$ der Radius von $K$, so wird

$r+s=\sqrt{s^{2}+\left|\zeta_{2}\right|^{2}}+s>2 s>1$, weil $s>\frac{1}{2}$, d. h. $K$ umschließt den Punkt - 1 der $\zeta$-Ebene, $C$ den Nullpunkt der $z$-Ebene.

Die Abschätzung des Beitrages über $C$ ist derjenigen über $C_{2}$ im Fall $x<4 n$ ganz analog; auf $K$ ist dabei zu setzen $\zeta=-s-r e^{-i \tau}$, wo die Bedeutung von $\tau$ aus Fig. 5 zu ersehen ist. Da $s=\frac{n}{\left|z_{0}\right|}<1$, so ist der Radius $r$ von $K$ beschränkt. Es existieren also auch hier 2 positive Konstanten $M$ und $\delta$ derart, daß

$$
\left|\frac{1}{2 \pi i} \int_{C} F(z) \frac{d z}{z}\right|<M\left|F\left(z_{0}\right)\right| e^{-\delta\left|a z_{0}\right|} .
$$


$\S$ 11. Die Einführung der Hilfsgröße $\beta$ durch

$$
\operatorname{ch} \beta=\frac{1}{2} \sqrt{\frac{x}{n}}, \quad(0<\beta<\infty)
$$

ergibt die Ausdrücke

$$
\begin{aligned}
& z_{0}=-2 n \operatorname{ch} \beta(\operatorname{ch} \beta-\operatorname{sh} \beta), 1+\frac{x}{z_{0}}=-(\operatorname{ch} 2 \beta+\operatorname{sh} 2 \beta), \\
& a=\operatorname{sh} \beta(\operatorname{ch} \beta-\operatorname{sh} \beta) .
\end{aligned}
$$

Setzt man sie in (23) ein, so resultiert die Formel (12) der Einleitung.

\section{Kapitel.}

\section{Der Fall $\boldsymbol{x}<0 ; \boldsymbol{n}|\boldsymbol{x}| \rightarrow \infty, \boldsymbol{n} \rightarrow \infty$.}

$\S 12$. Wie im II. Kapitel verlaufe der Integrationsweg nur durch den einen $\mathrm{Pa}$

$$
z_{0}=-\frac{x}{2}-\sqrt{\frac{x^{2}}{4}-n x} .
$$

Die Gestalt des Weges ist aus Fig. 4 ersichtlich.

Die Beziehungen

$$
\left|z_{0}\right|=-z_{0}=\frac{2 n}{1+\sqrt{1-\frac{4 n}{x}}}<n, \frac{1}{2}<a=1+\frac{z_{0}}{2 n}<1
$$

zeigen, daß man für die Umgebung des Passes $z_{0}$ den gleichen Ausdruck erhält wie im Fall $x>4 n(\S 9)$.

\section{$\S$ 13. Abschätzung von $\boldsymbol{R}_{k}^{\prime}$.}

Auf Grund von $\frac{\left|z_{0}\right|}{n}<1$ folgt $-2 \lg (1-2 \zeta)$ als Majorante von $g(\zeta)$ $\left(|\zeta|<\frac{1}{2}\right)$, und $(1-2 \zeta)^{-(1+2|\xi|)}$ als solche der Funktion (8). Die weitere Abschätzung verläuft analog wie früher und man findet

$$
R_{k}^{\prime}=\mathrm{O}\left(\left|z_{0}\right|^{-\frac{k}{2}}\right)
$$




\section{\$14. Beitrag des abrigen Integrationsweges}

Durch die Punkte $\zeta_{2}$ und $\zeta_{3}$ der Fig. 5 legen wir einen Kreisbogen mit dem Zentrum $\zeta_{4}=-1$. Das Stück in der oberen Halbebene sei $K^{*}$ und sein Bild in der $z$-Ebene $C^{*}$. Dann setzen wir wieder

$\int_{C^{*}} F(z) \frac{d z}{z}=-H(o) \frac{H\left(\zeta_{2}\right)}{H(o)} \cdot \int_{K^{*}} \exp \left[\left|z_{0}\right|\left(\frac{1}{1+\zeta}-\frac{1}{1+\zeta_{2}}\right)\right]\left(\frac{\frac{n}{\left|z_{0}\right|}+\zeta}{\frac{n}{\left|z_{0}\right|}+\zeta_{2}}\right)^{n} \frac{d \zeta}{1+\zeta}$.

Auf $K^{*}$ ist $\zeta=-1-r e^{-i \tau}, \zeta_{2}=-1-r e^{-i \tau_{0}}$ und folglich

$$
\begin{aligned}
& \Re\left[\left|z_{0}\right|\left(\frac{1}{1+\zeta}-\frac{1}{1+\zeta_{2}}\right)\right]=-\frac{\left|z_{0}\right|}{r}\left(\cos \tau-\cos \tau_{0}\right) \leqq 0 \\
& \left|\frac{n}{\mid \frac{z_{0} \mid}{n}+\zeta}\right|^{2}=1-\frac{2 r\left(\frac{n}{\left|z_{0}\right|}-1\right)\left(\cos \tau-\cos \tau_{0}\right)}{\left|\frac{n}{\left|z_{0}\right|}+\zeta_{2}\right|^{2}} \leqq 1, \text { weil } \frac{n}{\left|z_{0}\right|}>1 \\
& |1+\zeta|=r .
\end{aligned}
$$

Somit wird

$$
\left|\frac{1}{2 \pi i} \int_{C^{\star}} F(z) \frac{d z}{z}\right|<\frac{1}{2}\left|F\left(z_{0}\right)\right| e^{-\delta^{\star}\left|z_{0}\right|,}
$$

wo $\delta^{*}$ eine positive Konstante ist.

Für den in der unteren Halbebene gelegenen Kurventeil gilt dieselbe Abschätzung.

$\S$ 15. Die Einführung der Hilfsgröße $\gamma$ durch

$$
\operatorname{sh}^{2}(2 \gamma)=-\frac{4 n}{x} \quad(0<\gamma<\infty)
$$

liefert die Ausdrücke

$$
z_{0}=-\frac{n}{c^{2} \gamma}, 1+\frac{x}{z_{0}}=c t h^{2} \gamma, a=\frac{c h 2 \gamma}{2 c h^{2} \gamma} .
$$

In Verbindung mit (23) ergeben sie die Formel (13) der Einleitung. 


\section{Der Fall $x \sim 4 n$.}

$\S$ 16. Für $x=4 n$ gehen vom Punkt $-\frac{x}{2} 3$ Sektoren mit den Zentriwinkeln $\frac{\pi}{3}$ aus, in welchen $|F(z)|<\left|F\left(-\frac{x}{2}\right)\right|$. Ihre Winkelhalbierenden haben die Richtungswinkel $\frac{\pi}{3},-\frac{\pi}{3}$ und $\pi$. In der Umgebung des Passes setze sich der Integrationsweg aus 2 zur reellen Axe symmetrischen Kreisbogenstücken $C^{\prime}$ und $C^{\prime \prime}$ zusammen, deren Tangenten im $\mathrm{Pa}$ die Argumente $\frac{\pi}{3}$ und $-\frac{\pi}{3}$ besitzen. Die Endpunkte von $C^{\prime}$ und $C^{\prime \prime}$ werden durch einen Kreisbogen miteinander verbunden. Die genauere Festlegung folgt später.

Ist $x \sim 4 n$, so sind die Pässe $z_{0}$ und $z_{1}$ benachbart, und wir wählen in der Umgebung dieser Punkte durch den Punkt $-\frac{x}{2}$ denselben aus $C^{\prime}$ und $C^{\prime \prime}$ bestehenden Integrationsweg, der also nicht durch die Pässe von $|F(z)|$ geht.

\section{§ 17. Umgebung der Pässe}

Die Substitutionen $z=-\frac{x}{2(1+\zeta)}, \quad \zeta=-\omega\left(\frac{x}{2}\right)^{-\frac{1}{3}}$ ergeben, wenn zur Abkürzung

$\varrho=\left(\frac{x}{2}\right)^{\frac{1}{3}}$ und $t=-\left(\frac{x}{2}\right)^{\frac{2}{3}}\left(1-\frac{4 n}{x}\right)=2 n\left(\frac{x}{2}\right)^{-\frac{1}{3}}\left(1-\frac{x}{4 n}\right)$

gesetzt wird:

$\frac{1}{2 \pi i} \int_{C^{\prime} C^{\prime \prime}} F^{\prime}(z) \frac{d z}{z}=\frac{1}{2 \pi i} \cdot \frac{F\left(\frac{x}{2}\right)}{\varrho} \int_{A^{\prime}} \exp \left[\frac{\omega \varrho^{2}}{1-\frac{\omega}{\varrho}}+\frac{\varrho\left(\varrho^{2}+t\right)}{2} l g\left(1-\frac{2 \omega}{\varrho}\right)\right] \frac{d \omega}{1-\frac{\omega}{\varrho}}$

$\Lambda^{\prime}$ und $\Lambda^{\prime \prime}$ bedeuten 2 vom Nullpunkt der $\omega$-Ebene ausgehende Strecken 
mit den Argumenten $-\frac{2 \pi}{3}$ und $\frac{2 \pi}{3}$. Ihre Länge sei $\Theta \cdot \frac{\varrho}{2}, 0<\Theta<1$, ihre Endpunkte seien $\omega^{\prime}$ und $\omega^{\prime \prime}$. Mit $C^{\prime}$ und $C^{\prime \prime}$ werden die Bilder von $\Lambda^{\prime}$ und $\Lambda^{\prime \prime}$ in der $z$-Ebene bezeichnet, ihre Endpunkte mit $z^{\prime}$ und $z^{\prime \prime}$.

Setzt man zur Abkürzung für das Integral der rechten Seite von (27) $2 i G(t, \varrho)$, so wird

$$
\frac{1}{2 \pi i} \int_{C^{\prime} C^{\prime \prime}} F(z) \frac{d z}{z}=\frac{F\left(-\frac{x}{2}\right)}{\pi \cdot \varrho} G(t, \varrho) .
$$

$G(t, \varrho)$ ist eine ganze Funktion von $t$ und läßt sich in eine für jeden Wert von $t$ konvergente Reihe entwickeln

wo

$$
G(t, \varrho)=\sum_{p=0}^{\infty} \frac{A_{p}}{p !} t^{p},
$$

$$
A_{p}=-\frac{i}{2}\left(\frac{\varrho}{2}\right)^{p} \int_{\Lambda^{\prime} A^{\prime \prime}} \exp \left\{\varrho^{3}\left[\frac{\frac{\omega}{\varrho}}{1-\frac{\omega}{\varrho}}+\frac{1}{2} \lg \left(1-\frac{2 \omega}{\varrho}\right)\right]\right\}\left[\lg \left(1-\frac{2 \omega}{\varrho}\right)\right]^{p} \frac{d \omega}{1-\frac{\omega}{\varrho}} .
$$

§ 18. Asymptotische Formel für $\boldsymbol{A}_{p}$.

Da längs $\Lambda^{\prime}$ und $\Lambda^{\prime \prime}|\omega| \leqq \Theta \cdot \frac{\varrho}{2}<\frac{\varrho}{2}$, so resultiert

$$
A_{p}=-\frac{i}{2}(-1)^{p} \int_{\Lambda^{\prime} \Lambda^{\prime \prime}} \frac{\left[h_{1}(\vartheta)\right]^{p}}{1-\vartheta} e^{\xi h_{2}\{(\vartheta)} \omega^{p} e^{-\frac{\omega^{3}}{3}} d \omega,
$$

wo $\vartheta=\frac{\omega}{\varrho}, \xi=\omega^{3}$ und $h_{1}(\vartheta), h_{2}(\vartheta)$ die Funktionen $\left(9^{\prime}\right)$ und $\left(9^{\prime \prime}\right)$ bedeuten. Führt man die Entwicklung (9) ein, so geht $A_{p}$ über in

$$
A_{p}=-\frac{i}{2}(-1)^{p} \int_{A^{\prime} A^{\prime \prime}} \omega^{p} e^{-\frac{\omega^{3}}{3}} \sum_{\lambda=0}^{k-1} \chi_{\lambda_{p}}\left(\omega^{3}\right)\left(\frac{\omega}{\varrho}\right)^{2} d \omega+{R^{\prime}}_{k},
$$

wo

$$
R_{k}^{\prime}=-\frac{i}{2}(-1)^{p} \int_{\Lambda^{\prime} \Lambda^{\prime \prime}} \omega^{p} e^{-\frac{\omega^{8}}{3}} \sum_{\lambda=k}^{\infty} \chi_{\lambda p}\left(\omega^{3}\right)\left(\frac{\omega}{\varrho}\right)^{\lambda} d \omega
$$


Auf $\Lambda^{\prime}$ setzen wir $\omega=\eta e^{-\frac{2 \pi i}{3}}$ und auf $\Lambda^{\prime \prime} \omega=\eta \cdot e^{\frac{2 \pi i}{3}}$.

Dann folgt

$A_{p}=\Im\left[(-1)^{p} e^{\frac{2 \pi i}{3}(p+1)} \int_{0}^{\Theta \cdot \frac{\rho}{2}} \eta^{p} e^{-\frac{\eta^{3}}{3}} \sum_{\lambda=0}^{k-1} \chi_{\lambda p}\left(\eta^{3}\right)\left(\frac{\eta \cdot e^{\frac{2 \pi i}{3}}}{\varrho}\right)^{\lambda} d \eta\right]+R_{k}^{\prime}$

Ersetzt man die obere Grenze $\Theta \cdot \frac{\varrho}{2}$ des Integrals durch $\infty$, so begeht man einen Fehler $R_{k}^{\prime \prime}$. Schreibt man für $\chi_{\lambda p}$ die Entwicklung (6), so führt dies auf die Berechnung von Integralen der Form

$$
\int_{0}^{\infty} e^{-\frac{\eta^{3}}{3}} \eta^{p+3 \mu+\lambda} d \eta, \text { die den Wert } 3^{\frac{p-2}{3}+\frac{\lambda}{3}+\mu} \Gamma\left(\frac{p+\lambda+1}{3}+\mu\right)
$$

haben. Schließlich ergibt sich

$A_{p}=(-1)^{p} 3^{\frac{p-2}{3}} \sum_{\lambda=0}^{k-1} \sum_{\mu=0}^{\lambda} \frac{b_{\lambda \mu}^{(p)} 3^{\frac{\lambda}{3}+\mu}}{\varrho^{\lambda}} \Gamma\left(\frac{p+\lambda+1}{3}+\mu\right) \sin \left(\frac{2(p+\lambda+1)}{3} \pi\right)$

$+R_{k}^{\prime}+R_{k}^{\prime \prime}$.

$\S$ 19. Abschätzung von $\boldsymbol{R}_{k}^{\prime}$ und $\boldsymbol{R}_{k}^{\prime \prime}$.

Die Funktion (9) hat die Majorante

$$
\begin{aligned}
(1-2 \vartheta)^{-(1+p+5|\xi|)=1} & +\left(1+\frac{p+5|\xi|}{1}\right)(2 \vartheta) \\
& +\left(1+\frac{p+5|\xi|}{1}\right)\left(1+\frac{p+5|\xi|}{2}\right)(2 \vartheta)^{2}+\ldots
\end{aligned}
$$

Daraus ergibt sich

$$
\left|\sum_{\lambda=k}^{\infty} \chi_{\lambda p}(\xi) \vartheta^{\lambda}\right| \leqq 2^{k}(1+p+5|\xi|)^{k}|\vartheta|^{k}(1-2|\vartheta|)^{-(1+p+5|\xi|)} .
$$

Da im Integrationsintervall $0 \leqq \eta \leqq \Theta \cdot \frac{\varrho}{2} \quad 1-\frac{2 \eta}{\varrho} \geqq 1-\Theta$, so folgt

$$
\left|R_{k}^{\prime}\right| \leqq\left(\frac{2}{\varrho}\right)^{k} \frac{1}{(1-\Theta)^{1+p}} \int_{0}^{\Theta \frac{\varrho}{2}} e^{-\frac{\eta^{3}}{3}}(1-\Theta)^{-5 \eta^{3}} \eta^{p+k}\left(1+p+5 \eta^{3}\right)^{k} d \eta
$$


Für hinreichend kleines $\Theta$ erhalten wir

$$
R_{k}^{\prime}=\mathrm{O}\left(\varrho^{-k}\right)=\mathrm{O}\left(x^{-\frac{k}{3}}\right) .
$$

$R_{k}^{\prime \prime}$ kann für genügend großes $\varrho$ gegenüber $R_{k}^{\prime}$ vernachlässigt werden.

\section{§ 20. Beitrag des übrigen Integrationsweges.}

Durch die den Punkten $\omega^{\prime}$ und $\omega^{\prime \prime}(\S 17)$ entsprechenden Punkte $\zeta^{\prime}$ und $\zeta^{\prime \prime}$ der $\zeta$-Ebene legen wir einen Kreisbogen mit dem Mittelpunkt $-\frac{1}{2}$. Es genügt dabei, den in der oberen Halbebene liegenden Teil $K^{*}$ zu betrachten. Sein Bild in der $z$-Ebene nennen wir $C^{*}$.

Wir setzen

$$
\frac{1}{2 \pi i} \int_{C^{*}} F(z) \frac{d z}{z}=-\frac{1}{2 \pi i} \frac{H(0)}{\varrho} \frac{H\left(\zeta^{\prime}\right)}{H(0)} \varrho \int_{\boldsymbol{K}^{*}} \frac{H(\zeta)}{H\left(\zeta^{\prime}\right)} \frac{d \zeta}{1+\zeta},
$$

wo $H(\zeta)=\exp \left[\frac{\varrho^{3}}{1+\zeta}+\frac{\varrho\left(\varrho^{2}+t\right)}{2} \lg (-1-2 \zeta)\right], \zeta^{\prime}=\frac{\Theta}{2} e^{i \frac{\pi}{3}}$.

Nun ist

$$
\begin{aligned}
& \left|\frac{H\left(\zeta^{\prime}\right)}{H(0)}\right|=\mid \exp \varrho^{3}\left[-\frac{1}{3}\left(\frac{\Theta}{2}\right)^{3}-\frac{\left(\frac{\Theta}{2}\right)^{4} e^{\frac{i \pi}{3}}}{1+\frac{\Theta}{2} e^{\frac{i \pi}{3}}}\right. \\
& \left.-\frac{1}{2}\left(1+\frac{t}{\varrho^{2}}\right) \Theta^{4} \sum_{v=4}^{\infty} \frac{(-1)^{\nu}}{v} e^{\frac{i \nu \pi}{3}} \Theta^{\prime-4}+\frac{t}{\varrho^{2}} \frac{\Theta}{2}\left(e^{\frac{i \pi}{3}}-\frac{\Theta}{2} e^{i \frac{2 \pi}{3}}-\frac{\Theta^{2}}{3}\right)\right]
\end{aligned}
$$

Dieser Ausdruck zeigt, daß man 2 positive Konstanten $d^{\prime}$ und $d^{\prime \prime}$ finden kann, so daß für $|t|<d^{\prime} \varrho^{2}$ und genügend kleines $\Theta$

$$
\left|\frac{H\left(\zeta^{\prime}\right)}{H(0)}\right|<e^{-d^{\prime \prime} \rho^{3}} .
$$

Die Abschätzung des Integrals über $K^{*}$ geschieht gleich wie in den beiden Fällen $x \gtrless 4 n$. Es gibt eine positive Konstante $N$, so daß

$$
\left|\int_{K^{*}} \frac{H(\zeta)}{H\left(\zeta^{\prime}\right)} \frac{d \zeta}{1+\zeta}\right|<N
$$


Für den Beitrag von $C^{*}$ ergibt sich also

$$
\frac{1}{2 \pi i} \int_{C^{*}} F(z) \frac{d z}{z}=\frac{F\left(-\frac{x}{2}\right)}{\varrho} \cdot \mathrm{O}\left(\varrho e^{-d^{\prime \prime} \varrho^{3}}\right)
$$

\section{Kapitel.}

\section{Der Fall $\boldsymbol{n}|\boldsymbol{x}|$ beschränkt, $\boldsymbol{n} \rightarrow \infty$.}

$\S 21$. Wir setzen zunächst $x>0$ voraus. Als Integrationsweg wählen wir den Kreis $C$ durch die Pässe $z_{0}$ und $z_{1}$, dessen Mittelpunkt mit dem Nullpunkt der $z$-Ebene zusammenfällt. Der Radius von $C$ ist also gleich $\sqrt{n x}$.

\section{$\S 22$. Integration über $C$.}

Die Substitution $z=-v \sqrt{n x}$ führt auf:

$$
\frac{L_{n}(x)}{n !}=\frac{1}{2 \pi i} \int_{K} \exp \left[v \sqrt{n x}+n l g\left(1-\frac{1}{v} \sqrt{\frac{x}{n}}\right)\right] \frac{d v}{v},
$$

wo das Integral im positiven Sinn über den Einheitskreis $K$ der $v$-Ebene $\mathrm{zu}$ erstrecken ist.

$l g\left(1-\frac{1}{v} \sqrt{\frac{x}{n}}\right)$ läßt sich in eine Reihe entwickeln; denn $|v|=1>\sqrt{\frac{x}{n}}$, weil $\sqrt{\frac{x}{n}} \rightarrow 0$.

Man findet

$$
\begin{aligned}
& \frac{L_{n}(x)}{n !}=\frac{1}{2 \pi i} \int_{\boldsymbol{K}} \exp \left[\sqrt{n x}\left(v-\frac{1}{v}\right)\right] \exp \left[\xi \sum_{v=2}^{\infty} \frac{\zeta^{v-1}}{v}\right] \frac{d v}{v}, \\
& \text { wo } \xi=-\frac{\sqrt{n x}}{v} \text { und } \zeta=\frac{1}{v} \sqrt{\frac{x}{n}} .
\end{aligned}
$$

Führt man in (35) die Entwicklung (10) ein, so resultiert

$$
\frac{L_{n}(x)}{n !}=\sum_{\lambda=0}^{k-1} \frac{1}{2 \pi} i \int_{K} \exp \left[\sqrt{n x}\left(v-\frac{1}{v}\right)\right] \psi_{\lambda}\left(-\frac{\sqrt{n x}}{v}\right)\left(\frac{1}{v} \sqrt{\frac{x}{n}}\right)^{\lambda} \frac{d v}{v}+R_{k},(36)
$$


wobei zur Abkürzung

$$
R_{k}=\frac{1}{2 \pi i} \int_{K} \exp \left[\sqrt{n x}\left(v-\frac{1}{v}\right)\right] \sum_{\lambda=k}^{\infty} \psi_{\lambda}\left(-\frac{\sqrt{n x}}{v}\right)\left(\frac{1}{v} \sqrt{\frac{x}{n}}\right)^{\lambda} \frac{d v}{v}
$$

gesetzt ist. Ersetzt man in (36) $\psi_{\lambda}$ durch die Entwicklung (7), so erhält $\operatorname{man}$

$\frac{L_{n}(x)}{n !}=\sum_{\lambda=0}^{k-1} \sum_{\mu=0}^{\lambda}(-1)^{\mu} c_{\lambda \mu} n^{-\frac{\lambda-\mu}{2}} x^{\frac{\lambda+\mu}{2}} J_{\lambda+\mu}(2 \sqrt{n x})+R_{k}$,

wo mit $J_{v}$ die Besselsche Funktion $v$. Ordnung bezeichnet ist.

\section{$\S$ 23. Abschätzung von $\boldsymbol{R}_{\boldsymbol{k}}$.}

Aus der Ungleichung

$$
\begin{aligned}
& \left|\sum_{\lambda=k}^{\infty} \psi_{\lambda}(\xi) \zeta^{\lambda}\right| \leqq(1+|\xi|)^{k}|\zeta|^{k}(1-|\zeta|)^{-1-|\xi|} \quad \text { folgt } \\
& \left|R_{k}\right| \leqq(1+\sqrt{n x})^{k}\left(\sqrt{\frac{x}{n}}\right)^{k}\left(1-\sqrt{\frac{x}{n}}\right)^{-1-\sqrt{n x}}
\end{aligned}
$$

wenn man beachtet, daß auf $K|v|=1$ und

$$
\Re\left[\sqrt{n x}\left(v-\frac{1}{v}\right)\right]=0 \text { ist. }
$$

Da nach Voraussetzung $x<\frac{B_{1}}{n}$ ist, wird also

$$
\begin{aligned}
& \left(1-\sqrt{\frac{x}{n}}\right)^{-1-\sqrt{n x}}<B_{2} \text { und } \\
& \left|R_{k}\right|<B_{3} n^{-k}
\end{aligned}
$$

wo $B_{1}, B_{2}, B_{3}$ geeignete positive Konstanten sind.

$\S 24$. Ist $x<0$, so wählt man als Integrationsweg den Kreis mit dem Nullpunkt als Mittelpunkt und dem Radius $\sqrt{n|x|}\left(z_{0} \sim-\sqrt{n|x|}\right)$. Die Substitution $z=-i v \sqrt{n|x|}$ zeigt dann, daß (37) und (38) auch in diesem Fall unverändert gelten.

(Eingegangen den 22. Mai 1934.) 


\section{Lebenslauf}

Ich wurde im Februar 1907 in Bonndorf (Baden) geboren. Ich besuchte die Primarschule in St. Gallen und Zürich. Nach zwei Jahren Sekundarschule trat ich in die kantonale Oberrealschule in Zürich ein und legte im Herbst 1925 die Maturitätsprüfung ab. Hierauf studierte ich an der Abteilung für Mathematik und Physik der Eidgenössischen Technischen Hochschule und erwarb im Februar 1930 das Diplom. Hernach war ich vier Jahre Assistent für höhere Mathematik an der E.T.H. und bin seit Frühjahr 1932 als Hilfslehrer an der Kantonsschule Zürich tätig.

Während der Ausarbeitung der vorliegenden Promotionsarbeit gewährte mir Herr Prof. Dr. M. Plancherel mannigfache wertvolle Unterstützung, wofür ich ihm meinen wärmsten Dank ausspreche.

E. Moecklin. 